\title{
ORIGINAL
}

\section{Evolving changes in mortality of 13,301 critically ill adult patients with COVID-19 over 8 months}

\author{
Pedro Kurtz ${ }^{1,2,3}$, Leonardo S. L. Bastos ${ }^{4}$, Leila F. Dantas ${ }^{4}$, Fernando G. Zampieri ${ }^{1,5,6}$, Marcio Soares ${ }^{1}$,
} Silvio Hamacher ${ }^{4}$, Jorge I. F. Salluh ${ }^{1,7}$ and Fernando A. Bozza ${ }^{1,8^{*}}$ (1)

(c) 2021 Springer-Verlag GmbH Germany, part of Springer Nature

\begin{abstract}
Purpose: Clinical characteristics and management of COVID-19 patients have evolved during the pandemic, potentially changing their outcomes. We analyzed the associations of changes in mortality rates with clinical profiles and respiratory support strategies in COVID-19 critically ill patients.
\end{abstract}

Methods: A multicenter cohort of RT-PCR-confirmed COVID-19 patients admitted at 126 Brazilian intensive care units between February $27^{\text {th }}$ and October $28^{\text {th }}, 2020$. Assessing temporal changes in deaths, we identified distinct time periods. We evaluated the association of characteristics and respiratory support strategies with 60-day in-hospital mortality using random-effects multivariable Cox regression with inverse probability weighting.

Results: Among the 13,301 confirmed-COVID-19 patients, 60-day in-hospital mortality was 13\%. Across four time periods identified, younger patients were progressively more common, non-invasive respiratory support was increasingly used, and the 60-day in-hospital mortality decreased in the last two periods. 4188 patients received advanced respiratory support (non-invasive or invasive), from which 42\% underwent only invasive mechanical ventilation, 37\% only non-invasive respiratory support and $21 \%$ failed non-invasive support and were intubated. After adjusting for organ dysfunction scores and premorbid conditions, we found that younger age, absence of frailty and the use of non-invasive respiratory support (NIRS) as first support strategy were independently associated with improved survival (hazard ratio for NIRS first [95\% confidence interval], 0.59 [0.54-0.65], $p<0.001$ ).

Conclusion: Age and mortality rates have declined over the first 8 months of the pandemic. The use of NIRS as the first respiratory support measure was associated with survival, but causal inference is limited by the observational nature of our data.

Keywords: Coronavirus, Respiratory support, In-hospital mortality, Respiratory support, Non-invasive ventilation

*Correspondence: bozza.fernando@gmail.com; fernando.bozza@ini. fiocruz.br

${ }^{1}$ D'Or Institute for Research and Education (IDOR), Rio de Janeiro, RJ, Brazil

Full author information is available at the end of the article

Pedro Kurtz and Leonardo S.L. Bastos contributed equally to this work.

\section{Introduction}

Months after the coronavirus disease 2019 (COVID-19) pandemic had spread across Asia and Europe, Brazil became a hotspot for the infection, with sustained transmission afterwards $[1,2]$. The general perception is that the proportion of severe cases declined as compared to the initial surge, with a younger population affected, resulting in lower case-fatality rates [3]. However, it is not

\section{实 Springer}


clear how these changes have impacted the in-hospital outcomes of severe cases or how improvements in the clinical management of these patients may have led to the decline in mortality rates.

Although therapeutic options for severe COVID-19 patients have been tested recently in large clinical trials, [4-7] general questions on supportive care, such as the best initial ventilatory strategy, are still controversial $[8$, 9]. No conclusive data from randomized trials on SARS, MERS, or COVID-19 is currently available to guide ventilation practices [10]. Moreover, concerns related to the risk of aerosol generation and contamination of healthcare workers limited recommendations of non-invasive respiratory support strategies at the beginning of the COVID-19 epidemic [11].

Resource limitations to treat severe COVID-19 patients have been a concern for international health authorities, societies, and researchers [12,13]. Recently, we demonstrated the impact on clinical outcomes of the collapse of health systems during the COVID-19 epidemic in Brazilian regions, especially for patients requiring mechanical ventilation $[14,15]$. It is crucial to understand the clinical outcomes and factors contributing to mortality in different healthcare settings and, throughout the epidemic, to envision potential care improvement targets and optimal utilization of resources.

The present study analyzes the dynamic of severe COVID-19 admissions in 126 intensive care units (ICUs) from a middle-income country during the first 8 months of the epidemic. We hypothesized that variations in clinical characteristics, risk factors and resource use were related to the evolving changes in mortality. In a secondary hypothesis, we evaluated the association of initial respiratory support strategies with 60-day in-hospital mortality in patients with acute respiratory failure.

\section{Patients and methods}

\section{Study design and participants}

As the first confirmed SARS-CoV-2 infection in Brazil occurred on February $26^{\text {th }}$, this cohort study included patients admitted from February $27^{\text {th }}$ to October $28^{\text {th }}$, 2020, with vital status follow-up until December $27^{\text {th }}$. We included all adult patients with RT-PCR-confirmed SARS-CoV-2 infection admitted to the ICUs from an integrated hospital network (Rede D'Or São Luiz) present in eight Brazilian States. All patients analyzed had COVID-19 as their primary ICU admission diagnoses. One hundred and twenty-six ICUs from 42 hospitals prospectively collected data on every consecutive ICU admission (Supplementary Figure-sFigure 1 and Supplementary Table-sTable 1). Local Ethics Committee and the Brazilian National Ethics Committee (CAAE:

\section{Take-home message}

Age and frailty of critically ill patients have reduced over the first 8 months of the COVID-19 pandemic. Mortality rates have also progressively decreased in the last 5 months, after May 2020. Noninvasive respiratory support has been increasingly used and showed an association with improved 60-day survival in our study. Results of randomized trials are needed to confirm if strategies based on early non-invasive support for respiratory failure in severe COVID-19 improve outcomes.

$17,079,119.7 .0000 .5249)$ approved the study without the need for informed consent.

\section{Data collection and missing values}

Anonymized information from COVID-19 ICU-admitted patients was obtained from an electronic system used for benchmarking purposes (Epimed Monitor ${ }^{\circledR}$, Rio de Janeiro, Brazil) [16]. The database contains prospectively collected structured data of all ICU admissions. Characteristics at admission, including demographics, clinical diagnosis, comorbidities, source of admission, the Simplified Acute Physiology Score 3 (SAPS-3), the Sequential Organ Failure Assessment (SOFA) score, and the Modified Frailty Index (MFI), were considered for the analysis. We assessed the subsequent use of organ support, especially the initial advanced respiratory support implemented (non-invasive respiratory support strategies and invasive mechanical ventilation, NIRS and IMV, respectively), renal replacement therapy (RRT), vasopressors, and the hospital and ICU outcomes. NIRS was defined as either non-invasive positive pressure ventilation (NPPV) or high-flow nasal cannula (HFNC). Preparedness measures to absorb the surge of COVID-19 patients included the cancellation of elective surgeries, an increase in the number of ICU beds, and the implementation of care pathways for those with respiratory failure. To evaluate ICU preparedness, we compared the average number of ICU beds and occupancy rates between a prepandemic period (October 2019 to January 2020) and the peak of simultaneous hospitalizations.

We did not perform value imputation for the primary analysis, and we reported the number of complete cases for each variable (Supplemental Methods-sMethods).

\section{Outcomes}

The primary outcome was 60-day in-hospital mortality. Secondary outcomes were in-hospital and ICU mortality, as well as hospital and ICU length-of-stays (LOS).

\section{Statistical analysis}

We used median and interquartile range (IQR) or mean and standard deviation (SD) for quantitative variables, 
and frequencies and proportions for categories. We assessed the temporal dynamic of ICU hospitalizations and respiratory support utilization. We stratified our study population in time periods based on the daily number of ICU deaths using a method for evaluating structural changes in time series (e.g., inflection-point or change in trend; sMethods). Using linear models, this method identifies "breakpoints" in which a significant change in the curve's behavior (e.g., inflection-point or change in trend) occurred [17].

We compared clinical characteristics, organ support, and the use of NIRS or IMV as the first respiratory support measure, across the defined time periods. Amongst the subset of patients that required advanced respiratory support (NIRS and/or IMV), we performed univariate analyses of 60-day in-hospital mortality using KaplanMeier (KM) survival curves. We considered age (categorized in decades with $<40$ years as the reference), frailty (categorized as non-frail, pre-frail, and frail based on the MFI), and the initial respiratory support (NIRS first and IMV first), as the variables of clinical relevance, along with the time periods previously estimated. Differences among survival curves were evaluated with the log-rank test (confidence level: 0.05).

We evaluated the associations between the previously described variables with the 60-day in-hospital mortality in the subset of patients that required advanced respiratory support. We used a random-effects multivariable Cox proportional hazards model where the hazard is death. Due to the different case-mix among the hospitals, we considered the hospital variable as a source of random variability (random intercept) and adjusted the variables by the identified time periods. We estimated the hazard ratio (HR) and its corresponding 95\% confidence interval for each variable. To account for the nonrandomized allocation of respiratory support strategies, we used propensity-score-derived inverse-probability treatment weighting (IPTW) in the multivariable Cox model. Propensity scores were estimated using multivariable logistic regression model with the first respiratory support as the response variable $[18,19]$ (sMethods). Starting from a full multivariable model, we used a backward elimination process using $p$ values in combination with goodnessof-fit measures (Akaike information criteria [AIC] and Bayesian information criteria $[\mathrm{BIC}]$ ) to estimate the final model.

We also performed two sensitivity analyses to evaluate the robustness of our results. First, we built the final models using two alternative propensity-score-based methods: the Standardized Mortality Ratio (SMR)weighting and IPTW excluding patients with propensity scores outside of the $95 \%$ percentile [20]. Second, we performed the multivariable model including only patients with available data on $\mathrm{PaO} 2 / \mathrm{FiO} 2$ ratio (with adjustment for this variable) and reported another model estimating missing values of lung injury severity with multiple imputation using chained equations [21].

We performed all analyses in R 4.0.2 (more details in sMethods).

\section{Results}

From February $27^{\text {th }}$, to October $28^{\text {th }}, 2020$, a total of 61,471 consecutive adult ICU admissions occurred in 42 hospitals and 126 ICUs that prospectively collected data. Out of those, 13,301 (22\%) were patients with confirmed COVID-19 diagnosis, of which 4188 (31\%) had respiratory failure requiring advanced respiratory support (NIRS or IMV) (sFigure 2). The overall median age was 54 years (IQR:[41, 69]), with 39\% $(5,250 / 13,301)$ being 60 years or older and $42 \%$ women (Table 1). 13\% of patients were frail $(\mathrm{MFI} \geq 3)$, and $68 \%$ presented at least one comorbidity (sTable 4). Among patients with $\mathrm{PaO}_{2} / \mathrm{FiO}_{2}$ ratio available, almost half $(2112 / 4,649 ; 45 \%)$ presented moderate to severe lung injury $\left(\mathrm{PaO}_{2} / \mathrm{FiO}_{2}\right.$ ratio $\leq 200)$. The median ICU and hospital LOS were 5 days (IQR: [2-10]) and 8 days (IQR: [5-10]), respectively. Overall, 60-day in-hospital mortality was $13 \%$ $(1785 / 13,301)$.

Over time, since the first confirmed cases in late February and early March, a sharp rise in daily ICU admissions for COVID-19 was observed, with a subsequent increase in daily deaths (Fig. 1). This upward trend remained until May $13^{\text {th }}, 2020$, with a peak of 1,066 ICU-hospitalized patients in a single day. ICU mortality rate peaked $34 \%$ on May $24^{\text {th }}$. In comparison with the pre-pandemic period (October/2019-January/2020), ICU-bed availability increased $31 \%$ at the peak of ICU hospitalizations (sTable 2).

Based on the analysis of structural changes in the time series of the daily ICU deaths curve (Fig. 1, panel C), three breakpoints were identified, and four time periods were defined to stratify our population. Patient's characteristics and outcomes per period are described in Table 1. 60-day in-hospital mortality rates were: Period 1, 17\%; Period 2, 18\%, Period 3, 10\%; Period 4, 9.6\%. Patients in Periods 1 and 2 were older and more frequently frail. Clinical severity was highest in Period 2 (median SAPS3, 44 IQR: [39, 54]), while the need for vasopressors and RRT were worse in Period 1 (22\% and 12\%, respectively). Regarding modes of advanced respiratory support, we observed a progressive increase in the use of NIRS across the four time periods (Period 1, 8.3\%; Period 2, 16\%, Period 3, 20\%, and Period 4, 25\%).

Respiratory failure requiring advanced respiratory support occurred in 4,188 out of 13,301 (31\%) patients (sTable 3). Of these, 1765 (42\%) underwent invasive 
Table 1 Clinical characteristics and outcomes of 13,301 critically ill COVID-19 patients, total and by periods

\begin{tabular}{|c|c|c|c|c|c|}
\hline Characteristics & $\begin{array}{l}\text { Total } \\
n=13,301\end{array}$ & $\begin{array}{l}\text { Period } 1 \\
n=2184\end{array}$ & $\begin{array}{l}\text { Period } 2 \\
n=3536\end{array}$ & $\begin{array}{l}\text { Period } 3 \\
n=3938\end{array}$ & $\begin{array}{l}\text { Period } 4 \\
n=3643\end{array}$ \\
\hline Age, median (IQR) & $54(41,69)$ & $55(43,70)$ & $57(43,73)$ & $51(40,66)$ & $53(41,67)$ \\
\hline$<40$ & $2832(21 \%)$ & $421(19 \%)$ & $642(18 \%)$ & $943(24 \%)$ & $826(23 \%)$ \\
\hline $40-49$ & $2636(20 \%)$ & $421(19 \%)$ & $639(18 \%)$ & $853(22 \%)$ & $723(20 \%)$ \\
\hline $50-59$ & $2583(19 \%)$ & $433(20 \%)$ & 665 (19\%) & 769 (20\%) & 716 (20\%) \\
\hline $60-69$ & $2088(16 \%)$ & $354(16 \%)$ & $541(15 \%)$ & $558(14 \%)$ & $635(17 \%)$ \\
\hline $70-79$ & $1502(11 \%)$ & 275 (13\%) & $443(13 \%)$ & $414(11 \%)$ & $370(10 \%)$ \\
\hline$\geq 80$ & $1660(12 \%)$ & $280(13 \%)$ & $606(17 \%)$ & $401(10 \%)$ & $373(10 \%)$ \\
\hline \multicolumn{6}{|l|}{ Sex, No. (\%) } \\
\hline Female & $5549(42 \%)$ & $861(39 \%)$ & $1482(42 \%)$ & $1674(43 \%)$ & $1532(42 \%)$ \\
\hline Male & $7752(58 \%)$ & $1323(61 \%)$ & $2054(58 \%)$ & $2264(57 \%)$ & $2111(58 \%)$ \\
\hline Admissions from emergency department & $10,240(77 \%)$ & $1576(72 \%)$ & $2479(70 \%)$ & $3236(82 \%)$ & $2949(81 \%)$ \\
\hline \multicolumn{6}{|l|}{ Modified Frailty Index (MFI) } \\
\hline Mean (SD) & $1.07(1.25)$ & $1.13(1.25)$ & $1.26(1.33)$ & $0.96(1.20)$ & $0.96(1.19)$ \\
\hline Median (IQR) & $1(0,2)$ & $1(0,2)$ & $1(0,2)$ & $1(0,2)$ & $1(0,2)$ \\
\hline Non-frail (MFI=0) & $5860(44 \%)$ & $914(42 \%)$ & $1335(38 \%)$ & $1884(48 \%)$ & $1727(47 \%)$ \\
\hline Pre-frail (MFI=1-2) & $5717(43 \%)$ & $954(44 \%)$ & $1605(45 \%)$ & $1620(41 \%)$ & $1538(42 \%)$ \\
\hline Frail $(\mathrm{MFI}>=3)$ & $1724(13 \%)$ & $316(14 \%)$ & $596(17 \%)$ & $434(11 \%)$ & $378(10 \%)$ \\
\hline SAPS-3, median (IQR) & $42(37,50)$ & $43(37,52)$ & $44(39,54)$ & $41(37,48)$ & $42(38,49)$ \\
\hline SOFA, median (IQR) & $0(0,2)$ & $1(0,3)$ & $1(0,3)$ & $0(0,2)$ & $0(0,2)$ \\
\hline $\mathrm{PaO}_{2} / \mathrm{FiO}_{2}[\mathrm{n}=4,649]$ & $221(108,357)$ & $202(102,314)$ & $217(110,352)$ & $239(110,414)$ & $224(110,357)$ \\
\hline Normal $(>300)$ & $1580(34 \%)$ & $238(27 \%)$ & $475(32 \%)$ & $501(40 \%)$ & $366(35 \%)$ \\
\hline Mild (201-300) & $957(21 \%)$ & $200(23 \%)$ & $310(21 \%)$ & $217(17 \%)$ & $230(22 \%)$ \\
\hline Moderate (101-200) & $1015(22 \%)$ & 217 (25\%) & $352(24 \%)$ & $238(19 \%)$ & $208(20 \%)$ \\
\hline Severe $(\leq 100)$ & 1097 (24\%) & $216(25 \%)$ & $339(23 \%)$ & $293(23 \%)$ & $249(24 \%)$ \\
\hline Oxygen support, No. (\%) & $9113(69 \%)$ & $1457(67 \%)$ & $2272(64 \%)$ & $2880(73 \%)$ & $2504(69 \%)$ \\
\hline Advanced respiratory support, No. (\%) & $4188(31 \%)$ & $727(33 \%)$ & $1264(36 \%)$ & $1058(27 \%)$ & $1139(31 \%)$ \\
\hline Non-invasive respiratory support (NIRS) & $2423(18 \%)$ & $182(8.3 \%)$ & $567(16 \%)$ & $772(20 \%)$ & $902(25 \%)$ \\
\hline Only NPPV & $2061(85 \%)$ & $168(92 \%)$ & $519(92 \%)$ & $659(85 \%)$ & 715 (79\%) \\
\hline Only HFNC & $136(5.6 \%)$ & $8(4.4 \%)$ & $26(4.6 \%)$ & $48(6.2 \%)$ & $54(6.0 \%)$ \\
\hline Both & $226(9.3 \%)$ & $6(3.3 \%)$ & $22(3.9 \%)$ & $65(8.4 \%)$ & $133(15 \%)$ \\
\hline Only NIRS & $1558(12 \%)$ & $84(3.8 \%)$ & $308(8.7 \%)$ & $513(13 \%)$ & $653(18 \%)$ \\
\hline NIRS failure & $865(6.5 \%)$ & $98(4.5 \%)$ & $259(7.3 \%)$ & $259(6.6 \%)$ & $249(6.8 \%)$ \\
\hline Only invasive mechanical ventilation (IMV) & $1765(13 \%)$ & $545(25 \%)$ & $697(20 \%)$ & $286(7.3 \%)$ & $237(6.5 \%)$ \\
\hline Vasopressor, No. (\%) & $1986(15 \%)$ & $476(22 \%)$ & $735(21 \%)$ & $402(10 \%)$ & $373(10 \%)$ \\
\hline Renal replacement therapy, No. (\%) & $989(7.4 \%)$ & $256(12 \%)$ & $367(10 \%)$ & $215(5.5 \%)$ & $151(4.1 \%)$ \\
\hline \multicolumn{6}{|l|}{ Length-of-stay (LOS), Median (IQR) } \\
\hline $\mathrm{ICU}[n=13,294]$ & $5(2,10)$ & $6(3,13)$ & $6(3,12)$ & $4(2,9)$ & $5(2,9)$ \\
\hline Hospital $[n=13,219]$ & $8(5,15)$ & $9(6,18)$ & $10(6,18)$ & $7(5,14)$ & $7(5,13)$ \\
\hline \multicolumn{6}{|l|}{ Hospitalizations with LOS $>7$ days } \\
\hline $\mathrm{ICU}[n=13,294]$ & $4660(35 \%)$ & $899(41 \%)$ & $1398(40 \%)$ & $1190(30 \%)$ & $1173(32 \%)$ \\
\hline Hospital $[n=13,219]$ & $7304(55 \%)$ & $1324(61 \%)$ & $2252(64 \%)$ & $1931(49 \%)$ & $1797(50 \%)$ \\
\hline 60-day in-hospital deaths, No. (\%) & $1785(13 \%)$ & $380(17 \%)$ & $649(18 \%)$ & $405(10 \%)$ & $351(9.6 \%)$ \\
\hline Only NIRS $[n=1,558]$ & $72(4.6 \%)$ & $6(7.1 \%)$ & $14(4.5 \%)$ & $22(4.3 \%)$ & $30(4.6 \%)$ \\
\hline NIRS failure $[n=865]$ & $444(51 \%)$ & $40(41 \%)$ & $125(48 \%)$ & $139(54 \%)$ & $140(56 \%)$ \\
\hline Only IMV $[n=1,765]$ & $1028(58 \%)$ & $285(52 \%)$ & $430(62 \%)$ & $177(62 \%)$ & $136(57 \%)$ \\
\hline ICU deaths, No. (\%) [ $n=13,294]$ & $1446(11 \%)$ & $321(15 \%)$ & $542(15 \%)$ & $317(8.1 \%)$ & $266(7.3 \%)$ \\
\hline In-hospital deaths, No. (\%) [ $n=13,219]$ & $1814(14 \%)$ & $385(18 \%)$ & $662(19 \%)$ & $412(11 \%)$ & $355(9.8 \%)$ \\
\hline Ongoing patients, No. (\%) & $82(0.6 \%)$ & $16(0.7 \%)$ & $26(0.7 \%)$ & $15(0.4 \%)$ & $25(0.7 \%)$ \\
\hline
\end{tabular}


Table 1 (continued)

*Period 1-February $27^{\text {th }}$ to April $25^{\text {th }}$; Period 2-April $26^{\text {th }}$ to June $6^{\text {th }} ;$ Period 3-June $7^{\text {th }}$ to August $10^{\text {th }}$; and Period 4-August $11^{\text {th }}$ to October $28^{\text {th }}$.

SD—standard deviation; IQR_-interquartile range; SAPS—Simplified Acute Physiology Score; SOFA—Sequential Organ Failure Assessment; NIRS—non-invasive respiratory support; NPPV—non-invasive positive pressure ventilation; HFNC—high-flow nasal cannula; IMV—invasive mechanical ventilation; ICU—intensive care unit

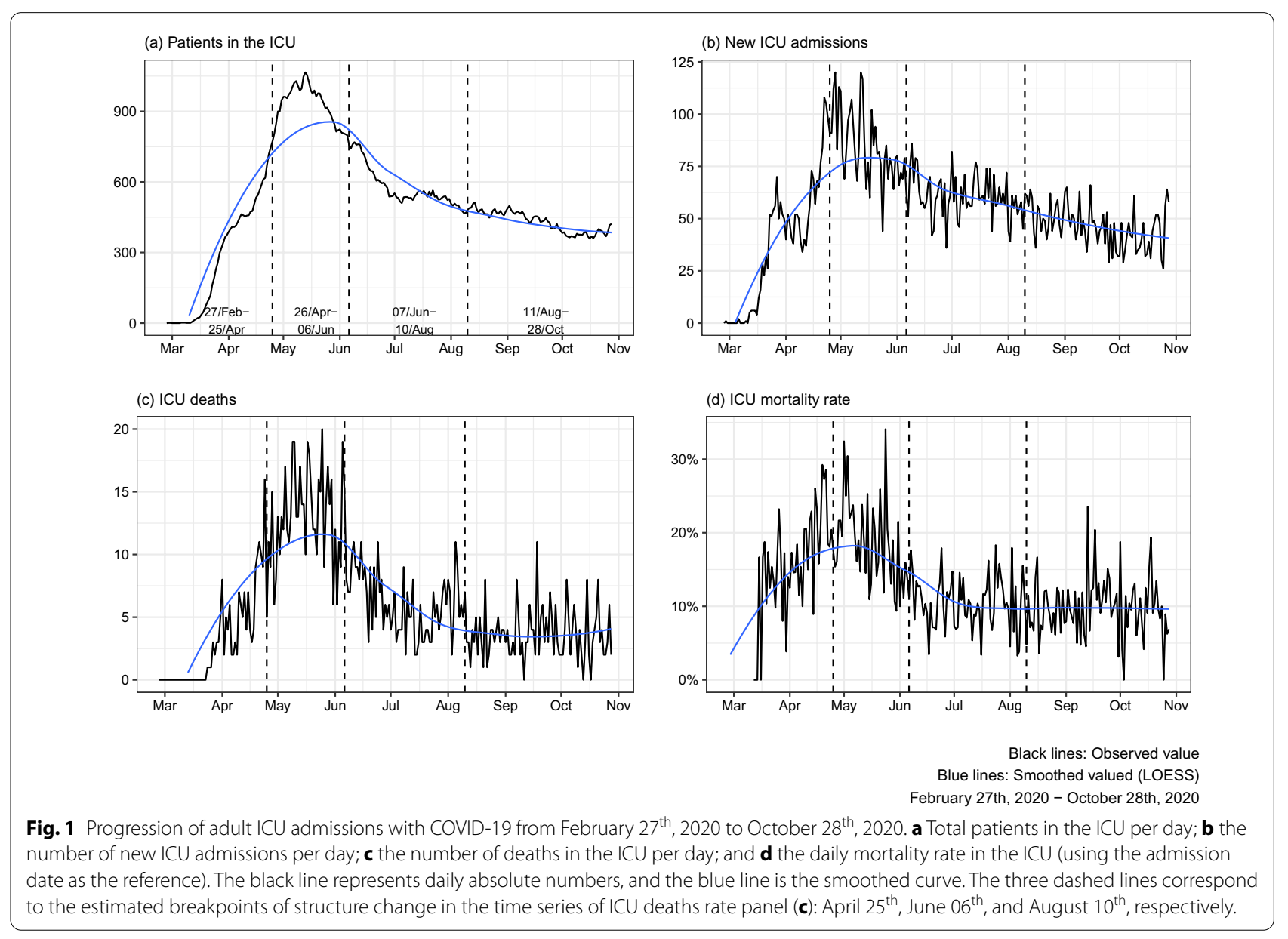

mechanical ventilation. In patients that received NIRS $(N=2423), 2061$ (85\%) used NPPV, 136 (6\%) HFNC, and $226(9 \%)$ received both treatment modes. 60-day in-hospital mortality was higher in those that underwent only IMV or failed NIRS as compared to those that required only NIRS ( 58 or $51 \%$ vs. $4.6 \%$; Table 2 ). In patients that underwent advanced respiratory support, the probability of survival was lowest in Periods 1 and 2 (Log-rank $p<0.0001$, Fig. 2, panel A). Stratification by age and frailty revealed progressively worse survival probabilities in patients older than 60 years and those that were pre-frail or frail, respectively (Log-rank $p<0.001$ in both, Fig. 2, panels $B$ and C). Regarding the initial strategies of respiratory support, the best survival probabilities were among patients that used NIRS as the first respiratory support measure as opposed to patients that were initially intubated (Fig. 2, panel D). Even patients with NIRS failure and subsequent intubation showed better survival probabilities compared to patients that first received IMV (sFigure 4).

We evaluated the association of clinical characteristics, risk factors and initial respiratory support strategies with 60-day in-hospital mortality in the subset of patients that underwent advanced respiratory support. We estimated a random-effects multivariable Cox model with IPTW (Fig. 3; sTable 5; sFigures 4-6). We found that older age (60-69 years, HR [95\% CI]: 1.47 [1.20-1.80], $p<0.001 ; 70-79$ years, HR [95\% CI]: 1.71 [1.38-2.10], $p<0.001 ; \geq 80$ years, HR [95\% CI]: 2.75 [2.21-3.41], $p<0.001$ ), and the presence of frailty (MFI $\geq 3, \mathrm{HR}[95 \%$ $\mathrm{CI}]: 1.38$ [1.15-1.64], $p<0.001)$ was independently associated with worse 60 -day survival. Moreover, the use 
Table 2 Characteristics and outcomes of critically ill patients stratified by advanced respiratory support

\begin{tabular}{|c|c|c|c|c|}
\hline Characteristics & $\begin{array}{l}\text { Total } \\
n=4,188\end{array}$ & $\begin{array}{r}\text { NIRS only } \\
n=1,558\end{array}$ & $\begin{array}{l}\text { NIRS failure } \\
n=865\end{array}$ & $\begin{array}{l}\text { IMV } \\
n=1,765\end{array}$ \\
\hline Age, median (IQR) & $63(49,76)$ & $55(43,67)$ & $65(53,77)$ & $68(54,80)$ \\
\hline$<40$ & $482(12 \%)$ & $286(18 \%)$ & $65(7.5 \%)$ & $131(7.4 \%)$ \\
\hline $40-49$ & $601(14 \%)$ & $307(20 \%)$ & $105(12 \%)$ & $189(11 \%)$ \\
\hline $50-59$ & $779(19 \%)$ & $376(24 \%)$ & $143(17 \%)$ & $260(15 \%)$ \\
\hline $60-69$ & $840(20 \%)$ & $270(17 \%)$ & $227(26 \%)$ & $343(19 \%)$ \\
\hline $70-79$ & $696(17 \%)$ & $162(10 \%)$ & $143(17 \%)$ & $391(22 \%)$ \\
\hline$\geq 80$ & $790(19 \%)$ & $157(10 \%)$ & $182(21 \%)$ & $451(26 \%)$ \\
\hline \multicolumn{5}{|l|}{ Sex, No. (\%) } \\
\hline Female & $1516(36 \%)$ & $546(35 \%)$ & $305(35 \%)$ & $665(38 \%)$ \\
\hline Male & $2672(64 \%)$ & $1012(65 \%)$ & $560(65 \%)$ & $1100(62 \%)$ \\
\hline Admissions from emergency department & $2848(68 \%)$ & $1244(80 \%)$ & $581(67 \%)$ & $1023(58 \%)$ \\
\hline \multicolumn{5}{|l|}{ Modified Frailty Index (MFI) } \\
\hline Non-frail $(M F I=0)$ & $1164(28 \%)$ & $617(40 \%)$ & $199(23 \%)$ & $348(20 \%)$ \\
\hline Pre-frail (MFI=1-2) & $2128(51 \%)$ & $732(47 \%)$ & $459(53 \%)$ & $937(53 \%)$ \\
\hline Frail $(\mathrm{MFI}>=3)$ & $896(21 \%)$ & $209(13 \%)$ & $207(24 \%)$ & $480(27 \%)$ \\
\hline SAPS-3, median (IQR) & $50(42,61)$ & $43(39,51)$ & $54(45,66)$ & $55(46,67)$ \\
\hline$\leq 42$ & $1,165(28 \%)$ & $727(47 \%)$ & $147(17 \%)$ & $291(16 \%)$ \\
\hline $43-50$ & $982(23 \%)$ & $434(28 \%)$ & $198(23 \%)$ & $350(20 \%)$ \\
\hline $51-61$ & $1,034(25 \%)$ & $276(18 \%)$ & $242(28 \%)$ & $516(29 \%)$ \\
\hline$>61$ & $1,007(24 \%)$ & $121(7.8 \%)$ & $278(32 \%)$ & $608(34 \%)$ \\
\hline SOFA, median (IQR) & $2(0,5)$ & $1(0,2)$ & $3(1,7)$ & $4(1,8)$ \\
\hline Any comorbidities, No. (\%) & $3393(81 \%)$ & $1111(71 \%)$ & $754(87 \%)$ & $1528(87 \%)$ \\
\hline $\mathrm{PaO}_{2} / \mathrm{FiO}_{2}[n=1,963]$ & $170(94,279)$ & $216(89,329)$ & $142(90,233)$ & $172(101,273)$ \\
\hline Normal $(>300)$ & $431(22 \%)$ & $139(32 \%)$ & $75(15 \%)$ & $217(21 \%)$ \\
\hline Mild (201-300) & $385(20 \%)$ & $89(21 \%)$ & $91(18 \%)$ & $205(20 \%)$ \\
\hline Moderate (101-200) & $621(32 \%)$ & $78(18 \%)$ & $191(38 \%)$ & $352(34 \%)$ \\
\hline Severe $(\leq 100)$ & $526(27 \%)$ & $125(29 \%)$ & $146(29 \%)$ & $255(25 \%)$ \\
\hline \multicolumn{5}{|l|}{ Non-invasive respiratory support } \\
\hline Only NPPV & $2061(85 \%)$ & $1356(87 \%)$ & $705(82 \%)$ & - \\
\hline Only HFNC & $136(5.6 \%)$ & $87(5.6 \%)$ & $49(5.7 \%)$ & - \\
\hline Both & $226(9.3 \%)$ & $115(7.4 \%)$ & $111(13 \%)$ & - \\
\hline Vasopressor, No. (\%) & $1890(45 \%)$ & $60(3.9 \%)$ & $672(78 \%)$ & $1158(66 \%)$ \\
\hline Renal replacement therapy, No. (\%) & $896(21 \%)$ & $24(1.5 \%)$ & $278(32 \%)$ & $594(34 \%)$ \\
\hline \multicolumn{5}{|l|}{ Length-of-stay (LOS), median (IQR) } \\
\hline $\mathrm{ICU}[n=4,185]$ & $12(7,22)$ & $8(4,11)$ & $19(12,27)$ & $16(9,27)$ \\
\hline Hospital $[n=4,160]$ & $17(10,30)$ & $11(8,16)$ & $24(16,38)$ & $22(12,38)$ \\
\hline \multicolumn{5}{|l|}{ Hospitalizations with LOS $>7$ days } \\
\hline $\operatorname{ICU}[n=4,185]$ & $3011(72 \%)$ & $788(51 \%)$ & 787 (91\%) & $1436(81 \%)$ \\
\hline Hospital $[n=4,160]$ & $3496(84 \%)$ & $1166(75 \%)$ & $804(94 \%)$ & $1526(87 \%)$ \\
\hline Period 1 (February $27^{\text {th }}$ to April $25^{\text {th }}$ ) & $727(17 \%)$ & $84(5.4 \%)$ & $98(11 \%)$ & $545(31 \%)$ \\
\hline Period 2 (April $26^{\text {th }}$ to June $6^{\text {th }}$ ) & $1264(30 \%)$ & $308(20 \%)$ & $259(30 \%)$ & $697(39 \%)$ \\
\hline Period 3 (June $7^{\text {th }}$ to August $10^{\text {th }}$ ) & $1058(25 \%)$ & $513(33 \%)$ & $259(30 \%)$ & $286(16 \%)$ \\
\hline Period 4 (August $11^{\text {th }}$ to October $28^{\text {th }}$ ) & $1139(27 \%)$ & $653(42 \%)$ & $249(29 \%)$ & $237(13 \%)$ \\
\hline 60-day in-hospital deaths, No. (\%) & $1544(37 \%)$ & $72(4.6 \%)$ & $444(51 \%)$ & $1028(58 \%)$ \\
\hline ICU deaths, No. (\%) [ $n=13,294]$ & $1329(32 \%)$ & $47(3.0 \%)$ & $398(46 \%)$ & $884(50 \%)$ \\
\hline In-hospital deaths, No. (\%) [ $n=13,219]$ & $1572(38 \%)$ & $73(4.7 \%)$ & $457(53 \%)$ & $1042(59 \%)$ \\
\hline
\end{tabular}

SD—standard deviation; IQR—interquartile range; SAPS—Simplified Acute Physiology Score; SOFA—Sequential Organ Failure Assessment; NIRS—non-invasive respiratory support; NPPV—non-invasive positive pressure ventilation; HFNC—high-flow nasal cannula; IMV—invasive mechanical ventilation; ICU—intensive care unit. 
(A)

$$
\text { - Period } 1 \text { - Period } 2 \text { - Period } 3 \text { - Period } 4
$$
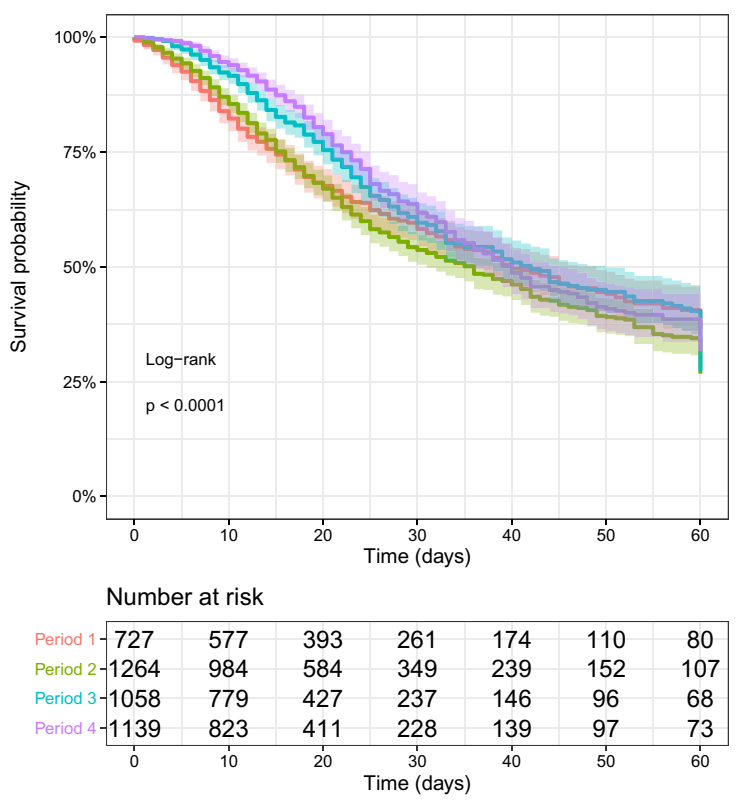

(C)
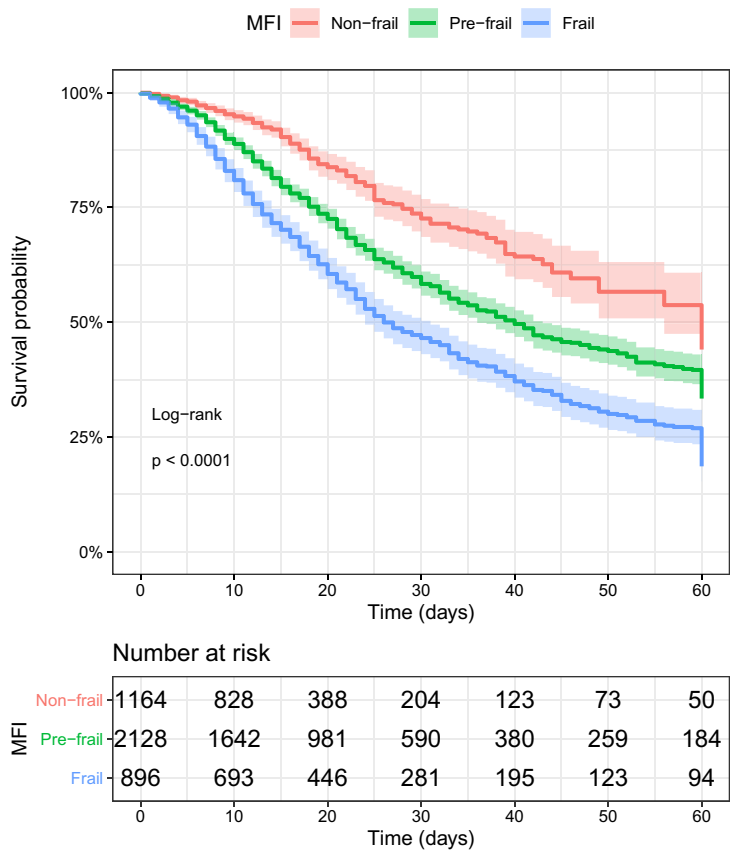

(B)

$$
\text { Age }=<40=40-49=50-59=60-69=70-79=>80
$$
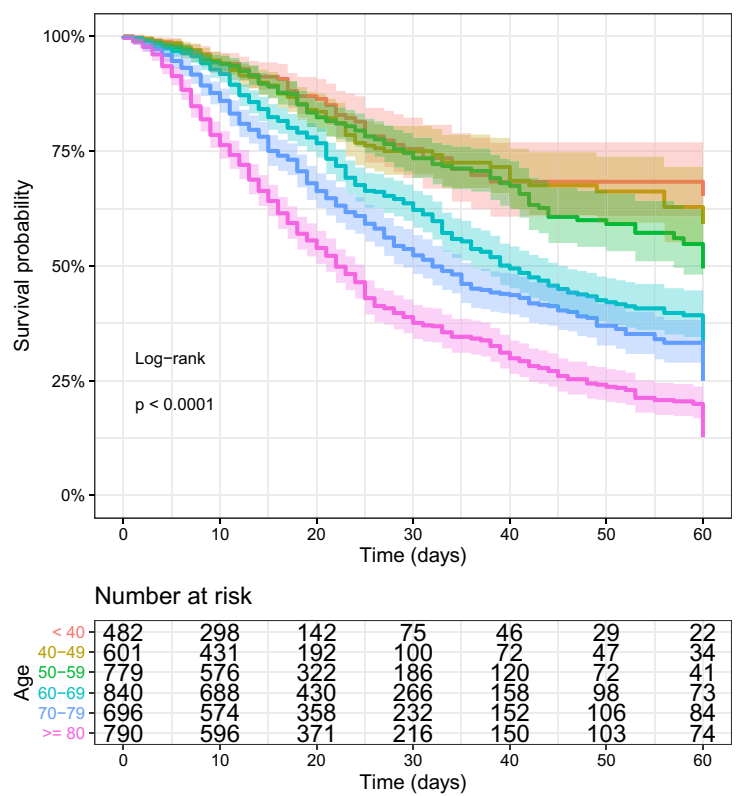

(D)
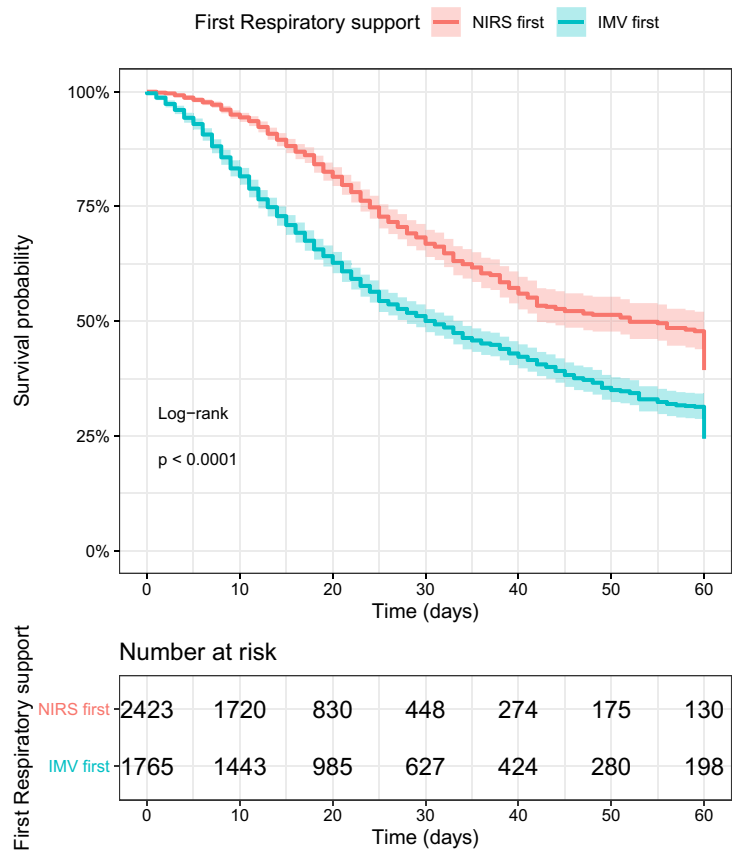

Fig. 2 Univariable survival curves (Kaplan-Meier) of factors related to the 60-day outcome in critically ill patients who underwent advanced respiratory support. $\mathbf{a}$ Time periods estimated with the breakpoints of structure change (Period 1: February $27^{\text {th }}$ to April $25^{\text {th }}$; Period 2: April $26^{\text {th }}$ to June $6^{\text {th }}$; Period 3: June $7^{\text {th }}$ to August $10^{\text {th }}$; Period 4: August $11^{\text {th }}$ to October $\left.28^{\text {th }}\right)$; b age $(<40,40-49,50-59,60-69,70-79$, and $\geq 80$; c Modified Frailty Index $(\mathrm{MFI})$ at the admission, with groups non-frail $(\mathrm{MFI}=0)$, pre-frail $(\mathrm{MFI}=1-2)$ and frail $(\mathrm{MFI} \geq 3)$; and $\mathbf{d}$ initial respiratory support considering noninvasive (NIRS first) invasive (IMV first). Differences among curves were assessed using the log-rank test with a confidence level of 0.05. 


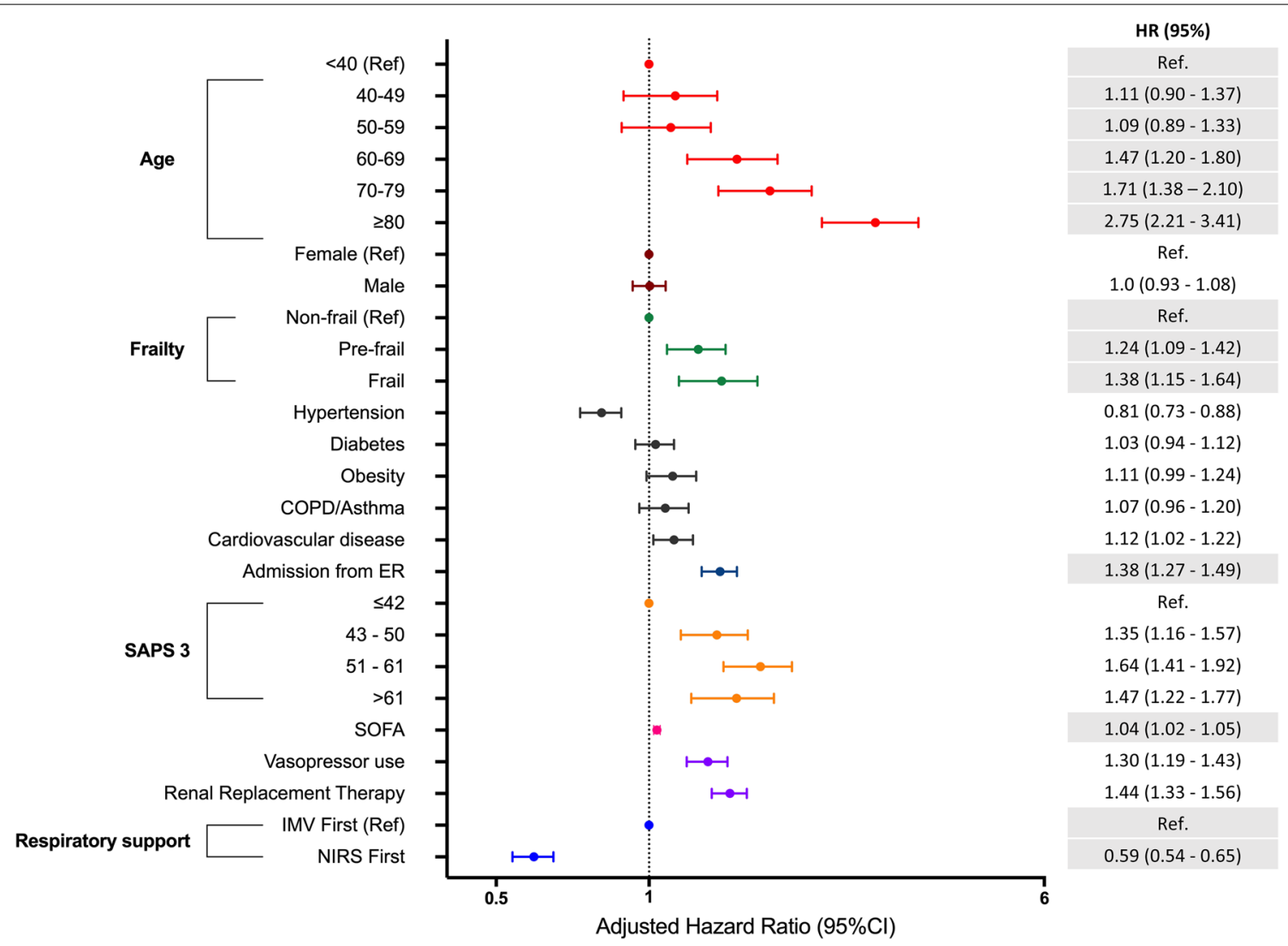

Fig. 3 Random-effects multivariable cox proportional hazards model to assess the association of clinical characteristics and initial respiratory support with 60-day mortality in patients who underwent advanced ventilatory support (NIRS and/or IMV), adjusted by the time-period of admission. The hospital was considered as the random intercept (standard deviation $=0.50$ ). To account for the non randomization, we used inverseprobability treatment weighting (IPTW) of propensity scores regarding the initial use of NIRS. We provide the hazard ratio (HR) for 60-day in-hospital mortality and its respective $95 \%$ confidence intervals for each variable

of NIRS, as the first respiratory support, was associated with improved survival over 60 days (HR [95\% CI]: 0.59 [0.54-0.65], $p<0.001$ ), after adjusting for the time periods, age, gender, frailty, SAPS-3 and SOFA scores, comorbidities, and source of admission (Fig. 3; sFigure 3). No significant multicollinearity was detected in the final model. All performed sensitivity analyses and alternative models demonstrated similar results in comparison with our primary analysis (sTables 7-13).

\section{Discussion}

In this large cohort of critically ill COVID-19 patients from South America, we showed the dynamic of the first 8 months of the epidemic and the evolving changes in clinical characteristics, respiratory support practices, and hospital mortality rates. Age and mortality rates declined over time after the peak in hospitalizations occurred, while daily ICU admissions reached a plateau. We further identified clinical predictors of 60-day in-hospital mortality, including increased age ( $>60$ years), the presence of frailty, multiple organ dysfunction, and the need for invasive mechanical ventilation. Finally, we observed an independent association between the increasing use of NIRS with improved survival in this population.

The dynamic reported in this work corresponded to the expansion of the outbreak over 8 months in the metropolitan areas of the country's southeast region $(\sim 70 \%$ of this sample). It also reflects a healthcare system that underwent preparedness with increases in ICU-bed and resource availability, which resulted in almost unrestricted access to ICU care. Our current findings contrast with recently published national data from the first 250 thousand patients hospitalized for COVID-19 in Brazil, where ICU mortality was $55 \%$. Even in the southeast region of the country-where the majority of our cohort was treated-authors showed that mortality in ICU patients was $49 \%$ and among those invasively ventilated it was $77 \%$ [15]. These differences may be due to early hospitalization, monitoring and good clinical practices performed in this hospital network. 
We analyzed the clinical characteristics and outcomes of critically ill COVID-19 patients admitted to 126 ICUs in 42 hospitals. We present complete data on 60-day inhospital outcomes (in addition to $99 \%$ of patients with available hospital mortality) from patients admitted over 8 months. To study the pandemic's temporal evolution, we analyzed structural changes in the mortality rate curve, which defined three breakpoints and four time periods. Survival was worst in the period when hospitalizations peaked, and patients were older and more frequently frail (Period 2). However, our results suggest that patients' characteristics alone do not explain the progressive reduction in mortality observed in Periods 3 and 4. Changes in management, such as the increased use of NIRS, were also related to improved survival rates in the subset of more severe patients that required advanced respiratory support.

In a European cohort from three countries, [22] investigators from the COVID-ICU group showed an overall decrease in 90-day mortality over time in critically ill patients from $42 \%$ in early March to $25 \%$ in late April 2020. Although they observed an increasing use of NIRS (mainly HFNC) and 41\% of patients received steroids, the associations of these interventions with mortality were not analyzed [23]. Overall, the use of steroids increased over time in the hospitals analyzed (sFigure 8). However, since we did not have individual data on treatment, we cannot exclude that changes in clinical management and other unmeasured interventions may have affected our findings of improved survival over time [24]. Nevertheless, we clearly demonstrated that NIRS (mainly NPPV in our cohort) was increasingly used over time during the study period and that, after adjusting for characteristics and time periods, it was associated with better survival.

In our study, $31 \%$ of patients required advanced ventilatory support (NIRS or IMV), which is lower than other multicenter ICU cohorts as well as data from the Brazilian ICU Registry [25]. Also, ventilated patients in our cohort were younger (median: 63 vs. 73 and 71 years in the UK and German cohorts, respectively), nevertheless, $81 \%$ presented comorbidities, and $21 \%$ were previously frail. Survival was progressively lower in patients older than 60 years and in those considered pre-frail or frail. We also observed a significantly higher NIRS utilization rate than other cohorts (Germany [26] 5\%, US [27] 1\%, Italy $[28]<10 \%)$. As expected, patients that underwent NIRS without subsequent intubation had improved survival as compared to those under invasive mechanical ventilation, but surprisingly, even patients that failed NIRS and were intubated also showed better survival compared to those that were intubated directly.

Concerns on biosafety aspects and potential intubation delays have limited the use of NIRS for respiratory failure early in the pandemic. However, NPPV and HFNC represent essential strategies in responding to respiratory emerging infections such as COVID-19, particularly in resource-limited settings, by optimizing critical care resources (i.e., invasive mechanical ventilation). In the absence of randomized trials, both from past severe viral infections and the current epidemic, our results that NIRS failure did not worsen mortality in comparison to those intubated directly, are reassuring for physicians using NIRS as an early option of ventilatory support for COVID-19. However, the potential benefit of noninvasive respiratory strategies in COVID-19-associated respiratory failure has yet to be determined by ongoing clinical trials (ISRCTN16912075 [29]).

The strengths of our study consist in being one of the largest multicenter cohorts of ICU-hospitalized patients with COVID-19, showing evolving mortality reductions in those critically ill. All patients had 60-day outcomes and detailed baseline severity of illness, comorbidities, frailty, organ dysfunction, and resource use information. Furthermore, we evaluated the association of respiratory support, especially NIRS, with 60-day mortality, which can inform future clinical trials and clinical practices for ICU patients. Potential limitations include: first, our sample may not reflect the epidemiology and practices in COVID-19 patients admitted to most Brazilian ICUs. Nonetheless, we showed data from a large network of hospitals with optimal preparedness and resource availability. Second, we cannot exclude that changes in clinical management and other unmeasured interventions may have affected survival over time, such as steroids, anticoagulation, and others. However, our models were adjusted for several clinically important covariates, including the four time periods over 8 months. Third, we analyzed NPPV and HFNC as one combined group of NIRS. We did not have specific data on NPPV-delivery methods (face mask or helmet) and only a small minority of patients underwent HFNC. These limitations prevent the interpretation of our results for any specific non-invasive ventilation mode. Fourth, we did not have imaging data on lung infiltrates or the diagnosis of viral pneumonia. However, all patients included in this analysis had a primary ICU admission diagnosis of COVID19 infection and required at least oxygen support in the ICU. Finally, although we had complete 60-day outcomes, the long-term follow-up and data on post-ICU quality of life or post-intensive care syndrome were unavailable.

\section{Conclusion}

In this large cohort of critically ill COVID-19 patients from South America, we demonstrated that, after a peak in hospitalizations occurred in May 2020, age and mortality rates have declined over the last 5 months of 
the epidemic. We also found an association between the use of non-invasive respiratory support and improved survival, even after accounting for age, frailty, organ failures, and conversion to invasive mechanical ventilation. These results, however, should be interpreted with caution, due to the observational nature of our data.

\section{Supplementary Information}

The online version contains supplementary material available at https://doi. org/10.1007/s00134-021-06388-0.

\begin{abstract}
Author details
1 D'Or Institute for Research and Education (IDOR), Rio de Janeiro, RJ, Brazil. ${ }^{2}$ Hospital Copa Star, Rio de Janeiro, RJ, Brazil. ${ }^{3}$ Paulo Niemeyer State Brain Institute (IECPN), Rio de Janeiro, RJ, Brazil. ${ }^{4}$ Department of Industrial Engineering (DEI), Pontifical Catholic University of Rio de Janeiro (PUC-Rio), Rio de Janeiro, RJ, Brazil. ${ }^{5}$ Research Institute, Hospital Do Coração (HCor), São Paulo, Brazil. ${ }^{6}$ Center for Epidemiological Research, Southern Denmark University, Odense, Denmark. ${ }^{7}$ Postgraduate Program of Internal Medicine, Federal University of Rio de Janeiro, Rio de Janeiro, UFRJ, Brazil. ${ }^{8}$ National Institute of Infectious Disease Evandro Chagas (INI), Oswaldo Cruz Foundation (FIOCRUZ), Rio de Janeiro, RJ, Brazil.
\end{abstract}

\section{Author contributions}

PK and LSLB contributed equally. FAB, PK, LSLB, MS, SH, and JIFS contributed to the study conception and design, and data interpretation. LSLB, PK, LFD, FGZ, and MS performed data processing and statistical analysis. FAB, LSLB, PK, LFD, $\mathrm{SH}$, and JIFS drafted the first version of the manuscript. FAB, SH, MS, and JIFS supervised the study. All authors had full access to data, participated in data interpretation, revised the manuscript, and approved the final version of the manuscript.

\section{Funding}

This study was supported by the National Council for Scientific and Technological Development (CNPq), the Coordination for the Improvement of Higher Education Personnel (CAPES)_Finance Code 001, Carlos Chagas Filho Foundation for Research Support of the State of Rio de Janeiro (FAPERJ), the Pontifical Catholic University of Rio de Janeiro, and by departmental funds from the D'Or Institute for Research and Education.

\section{Availability of data and material}

The data that support the findings of this study are available from the corresponding author, upon reasonable request.

\section{Code availability}

The programming code of data analysis is available in a GitHub repository (https://github.com/Islbastos/COVID19_ICU_Changes_Mortality).

\section{Declarations}

\section{Conflicts of interest}

The funders had no role in study design, data collection and analysis, decision to publish, or preparation of the manuscript. MS and JIFS are founders and equity shareholders of Epimed Solutions ${ }^{\circledR}$, which commercializes the Epimed Monitor System ${ }^{\circledR}$, a cloud-based software for ICU management and benchmarking. The other authors declare that they have no conflict of interest.

\section{Publisher's Note}

Springer Nature remains neutral with regard to jurisdictional claims in published maps and institutional affiliations.

Received: 11 December 2020 Accepted: 18 March 2021 Published online: 14 April 2021

\section{References}

1. Van Damme W, Dahake R, Delamou A et al (2020) The COVID-19 pandemic: diverse contexts; different epidemics—-how and why? BMJ Glob Health 5:e003098. https://doi.org/10.1136/bmjgh-2020-003098

2. Taylor L (2020) How Latin America is fighting covid-19, for better and worse. BMJ. https://doi.org/10.1136/bmj.m3319

3. Boehmer TK, DeVies J, Caruso E et al (2020) Changing Age Distribution of the COVID-19 Pandemic — United States, May-August 2020. MMWR Morb Mortal Wkly Rep 69:1404-1409. https://doi.org/10.1585/mmwr.mm693 9e1

4. Committee TW, for the REMAP-CAP Investigators, Angus DC, Derde L, et al (2020) Effect of Hydrocortisone on Mortality and Organ Support in Patients With Severe COVID-19: The REMAP-CAP COVID-19 Corticosteroid Domain Randomized Clinical Trial. JAMA. https://doi.org/10.1001/jama. 2020.17022

5. Tomazini BM, Maia IS, Cavalcanti AB et al (2020) Effect of dexamethasone on days alive and ventilator-free in patients with moderate or severe acute respiratory distress syndrome and COVID-19: the CoDEX randomized clinical trial. JAMA. https://doi.org/10.1001/jama.2020.17021

6. Wang Y, Zhang D, Du G et al (2020) Remdesivir in adults with severe COVID-19: a randomised, double-blind, placebo-controlled, multicentre trial. The Lancet 395:1569-1578. https://doi.org/10.1016/S0140-6736(20) 31022-9

7. Cao B, Wang Y, Wen D et al (2020) A trial of lopinavir-ritonavir in adults hospitalized with severe Covid-19. N Engl J Med 382:1787-1799. https:// doi.org/10.1056/NEJMoa2001282

8. Fan E, Beitler JR, Brochard L et al (2020) COVID-19-associated acute respiratory distress syndrome: is a different approach to management warranted? Lancet Respir Med 8:816-821. https://doi.org/10.1016/S22132600(20)30304-0

9. Winck JC, Ambrosino N (2020) COVID-19 pandemic and non invasive respiratory management: every Goliath needs a David. An evidence based evaluation of problems. Pulmonology 26:213-220. https://doi.org/ 10.1016/j.pulmoe.2020.04.013

10. Arabi YM, Fowler R, Hayden FG (2020) Critical care management of adults with community-acquired severe respiratory viral infection. Intensive Care Med 46:315-328. https://doi.org/10.1007/s00134-020-05943-5

11. Organization WH (2020) Clinical management of severe acute respiratory infection (SARI) when COVID-19 disease is suspected: interim guidance, 13 March 2020. World Health Organization

12. Dondorp AM, Hayat M, Aryal D et al (2020) Respiratory support in COVID19 patients, with a focus on resource-limited settings. Am J Trop Med Hyg 102:1191-1197. https://doi.org/10.4269/ajtmh.20-0283

13. Sopeyin A, Hornsey E, Okwor T et al (2020) Transmission risk of respiratory viruses in natural and mechanical ventilation environments: implications for SARS-CoV-2 transmission in Africa. BMJ Glob Health 5:e003522. https://doi.org/10.1136/bmjgh-2020-003522

14. Salluh JIF, Lisboa T, Bozza FA (2020) Challenges for the care delivery for critically ill COVID-19 patients in developing countries: the Brazilian perspective. Crit Care 24:593. https://doi.org/10.1186/s13054-020-03278-7

15. Ranzani OT, Bastos LSL, Gelli JGM et al (2021) Characterisation of the first 250000 hospital admissions for COVID-19 in Brazil: a retrospective analysis of nationwide data. Lancet Respir Med. https://doi.org/10.1016/ S2213-2600(20)30560-9

16. Zampieri FG, Soares M, Borges LP et al (2017) The Epimed Monitor ICU Database ${ }^{\circledR}$ : a cloud-based national registry for adult intensive care unit patients in Brazil. Revista Brasileira de Terapia Intensiva. https://doi.org/10. 5935/0103-507X.20170062

17. Zeileis A, Leisch F, Hornik K, Kleiber C (2002) strucchange: An R Package for Testing for Structural Change in Linear Regression Models. J Stat Soft. https://doi.org/10.18637/jss.v007.02

18. Greifer N (2020) Weightlt: Weighting for Covariate Balance in Observational Studies. https://cran.r-project.org/web/packages/Weightlt/index. html. Accessed 10 Jun 2020

19. Guo S, Fraser MW (2010) Propensity score analysis: statistical methods and applications. Sage Publications, Thousand Oaks, Calif

20. Kurth T, Walker AM, Glynn RJ et al (2006) Results of multivariable logistic regression, propensity matching, propensity adjustment, and propensitybased weighting under conditions of nonuniform effect. Am J Epidemiol 163:262-270. https://doi.org/10.1093/aje/kwj047 
21. van Buuren S, Groothuis-Oudshoorn K (2011) mice: multivariate imputation by chained equations in R. J Stat Soft. https://doi.org/10.18637/jss. v045.i03

22. COVID-ICU Group on behalf of the REVA Network and the COVID-ICU Investigators (2021) Clinical characteristics and day-90 outcomes of 4244 critically ill adults with COVID-19: a prospective cohort study. Intensive Care Med 47:60-73. https://doi.org/10.1007/s00134-020-06294-x

23. Management and Writing Committee and COVID-ICU Investigators, Schmidt M, Hajage D et al (2021) Clinical Characteristics and Day-90 Outcomes of 4,244 critically ill adults with COVID-19: a prospective cohort study. Intensive Care Med. https://doi.org/10.1007/s00134-020-06294-x

24. The WHO Rapid Evidence Appraisal for COVID-19 Therapies (REACT) Working Group, Sterne JAC, Murthy S et al (2020) Association between administration of systemic corticosteroids and mortality among critically III patients with COVID-19: a Meta-analysis. JAMA 324:1330. https://doi. org/10.1001/jama.2020.17023

25. UTIs brasileiras - Brazilian ICUs Brazilian ICUs - COVID-19 Benchmarking. In: COVID-19 Benchmarking. http://www.utisbrasileiras.com.br/en/covid19/benchmarking-covid-19/
26. Karagiannidis C, Mostert C, Hentschker C et al (2020) Case characteristics, resource use, and outcomes of 10021 patients with COVID-19 admitted to 920 German hospitals: an observational study. Lancet Respir Med. https://doi.org/10.1016/S2213-2600(20)30316-7

27. Gupta S, Hayek SS, Wang W et al (2020) Factors associated With death in critically III patients with coronavirus disease 2019 in the US. JAMA Intern Med. https://doi.org/10.1001/jamainternmed.2020.3596

28. Grasselli G, Greco M, Zanella A et al (2020) Risk factors associated with mortality among patients with COVID-19 in intensive care units in Lombardy, Italy. JAMA Intern Med. https://doi.org/10.1001/jamainternmed. 2020.3539

29. Perkins GD, Couper K, Connolly B et al (2020) RECOVERY- respiratory support: respiratory strategies for patients with suspected or proven COVID-19 respiratory failure; continuous positive airway pressure, highflow nasal oxygen, and standard care: a structured summary of a study protocol for a randomised controlled trial. Trials 21:687. https://doi.org/ 10.1186/s13063-020-04617-3 